\title{
A novel system for water soluble protein encapsulation with high efficiency: "Micelles enhanced" polyelectrolyte capsules
}

\author{
Xiaodong Li, ${ }^{1,2}$ Xiaohui Li, ${ }^{3}$ Jianxiang Zhang, ${ }^{4}$ Shifang Zhao, ${ }^{1}$ Jiacong Shen ${ }^{2}$ \\ ${ }^{1}$ Affiliated Stomatology Hospital, College of Medicine, Zhejiang University, \\ Hangzhou 310027, China \\ ${ }^{2}$ Department of Polymer Science and Engineering, Institute of Polymer Composite, Zhejiang University, \\ Hangzhou 310027, China \\ ${ }^{3}$ Department of New Drug Research Center, Faculty of Basic Medicine, Third Military Medical University, \\ Chongqing 400038, China \\ ${ }^{4}$ Department of Biologic and Materials Science, University of Michigan, Ann Arbor, Michigan 48109
}

Received 2 December 2006; revised 4 May 2007; accepted 22 May 2007

Published online 26 September 2007 in Wiley InterScience (www.interscience.wiley.com). DOI: 10.1002/jbm.a.31543

\begin{abstract}
Novel "micelles enhanced" polyelectrolyte (PE) capsules based on functional templates of hybrid calcium carbonate were fabricated. Evidences suggested that the structure of capsule wall was different from that of conventional PE capsules, and the wall permeability of these PE capsules changed significantly. Lysozyme, a positively charged protein in neutral solution, was studied as a model protein to be encapsulated into the "micelles enhanced" PE capsules. Confocal laser scanning microscope was used to observe the entrapping process in real time, while UV-Vis spectroscope and scanning force microscope measurements
\end{abstract}

suggested the high efficiency of encapsulation. In addition, the fluorescence recovery after photobleaching technique was employed to determine the existence form of deposited molecules. Further studies showed even negatively charged water-soluble peptides or proteins can be encapsulated into these hybrid capsules by modulating the $\mathrm{pH}$ value in bulk solution under its isoelectronic point as well. (C) 2007 Wiley Periodicals, Inc. J Biomed Mater Res 85A: 768-776, 2008

Key words: "micelles enhanced"; capsules; induced deposition; layer-by-layer; water soluble protein

\section{INTRODUCTION}

Layer-by-layer (LbL) assembly of oppositely charged polyelectrolytes (PE) on sacrificial core particles produces ultrathin PE-multilayer microcapsules after the core removal. ${ }^{1-4}$ A variety of different materials such as dyes, oxometalates, inorganic particles, and dendrimers in combination with linear PEs have been used to prepare multilayer microcapsules so that their physicochemical properties can be customized for various applications. ${ }^{5-8}$ Moreover, many attempts

Correspondence to: X. Li; e-mail: lixiaodong_yb@hotmail. com (or) X. Li; e-mail: xhl@mail.tmmu.com.cn

Contract grant sponsor: Natural Science Foundation of Zhejiang Province; contract grant number: 205183

Contract grant sponsor: Key National Natural Science Foundation of China; contract grant number: 50333020

Contract grant sponsor: National Basic Research Program of China (973 Program); contract grant number: 2005CB623902

(C) 2007 Wiley Periodicals, Inc. were made to encapsulate biomolecules into the PE capsules, such as enzymes and drugs, which could be made into a form of core template or be attached to core template particles prior to core decomposition so as to entrap them inside hollow capsules. ${ }^{9-11}$ Macromolecules can be encapsulated into preformed capsules either using in situ polymerization of monomers inside the capsules via a "ship in bottle" synthesis ${ }^{12}$ or by changing the wall permeability of the capsules by altering the bulk $\mathrm{pH} .{ }^{13,14}$ Actually, low efficiency encapsulation or complicated procedure of these methods was usually found. It was reported that water-soluble substances could spontaneously deposit into the melamine formaldehyde (MF) based capsules due to the presence of MF residues in the capsule interior. ${ }^{15}$ Because of the accidental amount of oligomer in the capsule interior, the encapsulation procedure cannot be well controlled for variable applications. ${ }^{16}$ Anyway, it provides a new visual angle for encapsulating guest molecules into PE capsules-designing functional templates to govern encapsulation and wall permeability of resulting PE capsules. 
In this study, novel "micelles enhanced" PE capsules based on functional core templates-hybrid calcium carbonate were fabricated according to our previous report. ${ }^{17}$ The induced accumulation of positively charged water soluble peptide and enzyme into these novel capsules was investigated in detail. By quantifying the concentration of lysozyme inside the capsule interior through UV-Vis measurements and observing the difference of the capsule wall thickness before and after incubation via scanning force microscope (SFM), it was found that this kind of capsules could entrap water soluble substances with high efficiency. Confocal laser scanning microscope (CLSM) was used to observe the entrapping process in real time. In addition, the fluorescence recovery after photobleaching (FRAP) technique was employed to determine the existence form of deposited molecules. Furthermore, albumin, a negatively charged protein in neutral solution, was also investigated as a model protein to be encapsulated into "micelles enhanced" capsules by modulating $\mathrm{pH}$ value of bulk solution.

\section{MATERIALS AND METHODS}

\section{Materials}

Amphiphilic block copolymer polystyrene- $b$-poly (acrylic acid) (PS- $b$-PAA, $M_{\mathrm{w}}=12,500$, the monomer molar ratio of styrene to acrylic acid in copolymer is about 2) was obtained from Rohm \& Hass. Poly (styrenesulfonate sodium salt) (PSS) $\left(M_{\mathrm{w}}=70,000\right)$ and poly (allylamine hydrochloride) $(\mathrm{PAH})\left(M_{\mathrm{w}}=70,000\right)$ were from Aldrich. Fluorescein isothiocynate (FITC) was purchased from Aldrich. Serum albumin $\left(M_{\mathrm{w}}=67,000\right)$ was purchased from Sigma. Lysozyme $\left(M_{\mathrm{w}}=14,300\right)$ was supplied by Shanghai Zhibo Chemical Corp. PAH and PSS were dissolved into $0.5 \mathrm{M}$ $\mathrm{NaCl}$ solution, respectively, and the concentration of these two PEs came up to $2 \mathrm{mg} / \mathrm{mL}$. FITC labeled lysozyme and albumin with molar ratio of lysozyme or albumin to FITC of 1:4 were separately prepared in our lab. All other reagents were commercially available and used as received.

\section{Fabrication of PSS/PAH multilayers on hybrid $\mathrm{CaCO}_{3}$ templates}

Hybrid microparticles were prepared according to the method reported in Ref. 17. PAH and PSS were absorbed on hybrid $\mathrm{CaCO}_{3}$ microparticles in turn. The first layer is PAH. After $10 \mathrm{~min}$ of adsorption for each polyeletrolyte, the hybrid templates were centrifuged and the supernatant was discarded. Then the precipitate was rinsed with $0.05 \mathrm{~mol} / \mathrm{L} \mathrm{NaCl}$ solution three times. The oppositely charged PE was then added. Each adsorption step followed by triple-rinse with $0.05 \mathrm{~mol} / \mathrm{L} \mathrm{NaCl}$ solution. After the adsorption of 12 layers, the hybrid cores were dissolved in trisodium EDTA $(\mathrm{pH}=7.3)$ for $30 \mathrm{~min}$ at agitation. The capsules were centrifuged, the supernatant was removed, and the capsules were resuspended in fresh tri- sodium EDTA. The washing procedure with tri-sodium EDTA was repeated four times. The resultant suspension of the formed microcapsules was washed four times with deionized water and stored in the centrifuge tubes.

\section{Entrapping positively charged lysozyme into capsules}

CLSM images were collected with a Zeiss laser scanning system (510) equipped with a $40 \times$ objective. FITC-lysozyme was used as fluorescence probe. Spontaneous loading of positively charged substances into hybrid capsules at the neutral $\mathrm{pH}$ was studied with real-time confocal microscopy. Three PE multilayers [(PEI/PSS)PEI] were predeposited on the glass slide to keep the PE capsules immobile on it. The capsules suspension was dropped on the glass slide in advance (about $20 \mu \mathrm{L}$ ). Using constant laser intensity and PMT gain, CLSM was preset to auto-collect images every $15 \mathrm{~s}$. Three microliter of probe solution $(40 \mu \mathrm{g} / \mathrm{mL})$ was added into the drop of capsules suspension, and the CLSM images were collected immediately. The fluorescence intensity from regions of interest inside the optically sectioned capsule was plotted versus time.

\section{Study of the existence form of the entrapped substances in the capsules}

As aforementioned, FITC-lysozyme filled capsules at the neutral $\mathrm{pH}$ value were fixed on the glass slide carrier with predeposited three PE multilayers [(PEI/PSS)PEI] in advance. The marked spots in FITC-lysozyme filled capsules were bleached for $1 \mathrm{~s}$ at maximum laser output at 488 $\mathrm{nm}$. The recovery images were recollected and the relative fluorescence intensity was calculated automatically by using Zeiss 510 software.

\section{Concentration determination of the entrapped substances inside the capsule interior}

The concentration of the capsules in suspension was measured by Flow Cytometer (Becton Dickinson, FACSORT, USA). FITC-lysozyme was used as a spectral probe. UV-Vis measurement was adopted to quantify the concentration of the encapsulated substances inside the capsules. Six samples of capsules suspension $(150 \mu \mathrm{L})$ were incubated with $400 \mu \mathrm{L}$ lysozyme solution with various concentrations ranging from 20,50, 100, 500, 1000 to $2000 \mu \mathrm{g} / \mathrm{mL}$. The supernatant after incubation for $2 \mathrm{~h}$ was diluted and FITC-lysozyme concentration was determined by the absorbance at $488 \mathrm{~nm}$. If the absorbance is above 1, the sample will be diluted to keep the absorbance versus concentration in linear range. The lysozyme concentration inside the capsule interior was calculated according to Eq. (1)

$$
\begin{gathered}
C=\frac{\Delta m}{v N \times(4 / 3) \pi r^{3}} \\
\Delta m=m_{0}-m_{1} \\
m_{1}=\alpha \times K I
\end{gathered}
$$

Where, $C$ is the mean concentration inside the capsule interior (neglecting the amount of lysozyme absorbed on the 


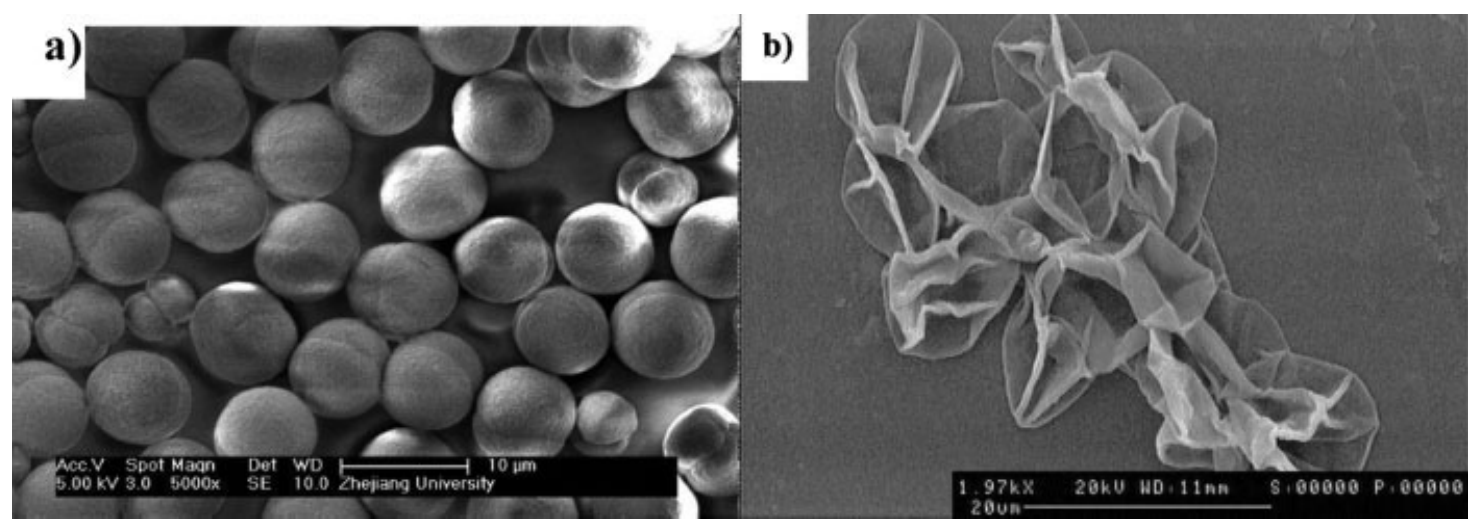

Figure 1. FESEM images of (a) the hybrid microparticles, and (b) "micelles enhanced" hybrid capsules.

outside PSS layer), $N$ is the concentration of the capsules in suspension $(\mathrm{N} / \mathrm{mL}), v$ is the volume of incubation solution $(\mathrm{mL}), r$ is the radius of capsules $(\mathrm{cm}), m_{0}$ is the initial mass of FITC-lysozyme in each of the six incubation solution samples $(\mu \mathrm{g}), K$ is the slope of calibration curve of FITC-lysozyme in water, $\alpha$ is the diluted times, $I$ is the absorbance of the lysozyme solution after incubation.

\section{Albumin encapsulation under different pH conditions}

Albumin encapsulation under different $\mathrm{pH}$ conditions was studied with real-time confocal microscopy. The drop of capsules suspension $(\mathrm{pH}=7)$ was fixed onto the glass slide carrier with pre-deposited three PE multilayers [(PEI/PSS)PEI] in advance $(20 \mu \mathrm{L})$. Three microliter of FITC-albumin solution $(40 \mu \mathrm{g} / \mathrm{mL})$ at the neutral $\mathrm{pH}$ value was dropped in the drop of capsules suspension. Using constant laser intensity and PMT gain, CLSM was set to auto-collect images every $15 \mathrm{~s}$. Three microliter of FITC-albumin in acidic solution $(\mathrm{pH}=3 ; 40 \mu \mathrm{g} / \mathrm{mL}$ ) was added into the capsules drop, and the CLSM images were collected immediately.

\section{Measurements}

The size and size distribution of the PE capsules was determined by coulter LS-230 laser particle size analyzer (Miami, USA). Field emission scanning electronic microscope (FESEM, SIRION-100, PHILLIPS) was used to observe the morphology of the particles after a gold-palladium layer was sputtered by E-1020 ion sputter for $120 \mathrm{~s}$. The samples were prepared by applying a drop of capsules suspension onto freshly cleaved mica, dried at room temperature and normal pressure. UV-Vis curves were obtained using a Perkin-Elmer UV-Vis Lambda 40 Spectrophotometer. SFM images were collected in air at $20-25^{\circ} \mathrm{C}$ using a Nanoscope III Multimode SFM (Digital Instruments, Santa Barbara, CA). The samples were prepared by applying a drop of the capsules solution onto freshly cleaved mica, dried at room temperature and normal pressure.

\section{RESULTS AND DISCUSSION}

The hybrid calcium carbonate microparticles with a mean size of $8.4 \mu \mathrm{m}$ were synthesized in the present of block copolymer PS- $b$-PAA according to the method reported in the Ref. 17. FESEM images in Figure 1(a) shows the morphology of the hybrid $\mathrm{CaCO}_{3}$ microparticles. These hybrid particles could serve as functional templates for fabricating PE capsules by LbL technique. Novel "micelles enhanced" PE capsules were thus obtained. The wall of the resulting capsules is significantly different from that of the conventional PE capsules with the same layers, ${ }^{18,19}$ which might lead to different permeability and encapsulation properties in contrast to conventional PE capsules.

\section{Study on lysozyme encapsulation into the "micelles enhanced" polyelectrolyte capsules}

Because of the presence of a large number of negatively charged micelles inside the capsules, the "micelles enhanced" PE capsules might have special properties of encapsulation. Here FITC-lysozyme was used as the model protein to study the encapsulation properties of "micelles enhanced" capsules. Induced deposition of the FITC-lysozyme was studied by CLSM as shown in Figure 2. After the addition of FITC-lysozyme, fluorescence intensity inside capsules increased gradually. Selected images of capsules at $0,60,180,360,600$, and $900 \mathrm{~s}$ during the loading process are presented in Figure 2(a-f). In addition, the line-scan of fluorescence intensity for a single microcapsule in inset of Figure 2 clearly showed the process of induced deposition. As shown in Figure 2, it can be observed clearly that the fluorescence intensity inside the capsule interior was significantly higher than that in the environment, indicating the higher FITC-lysozyme concentration inside the capsule interior compared with 

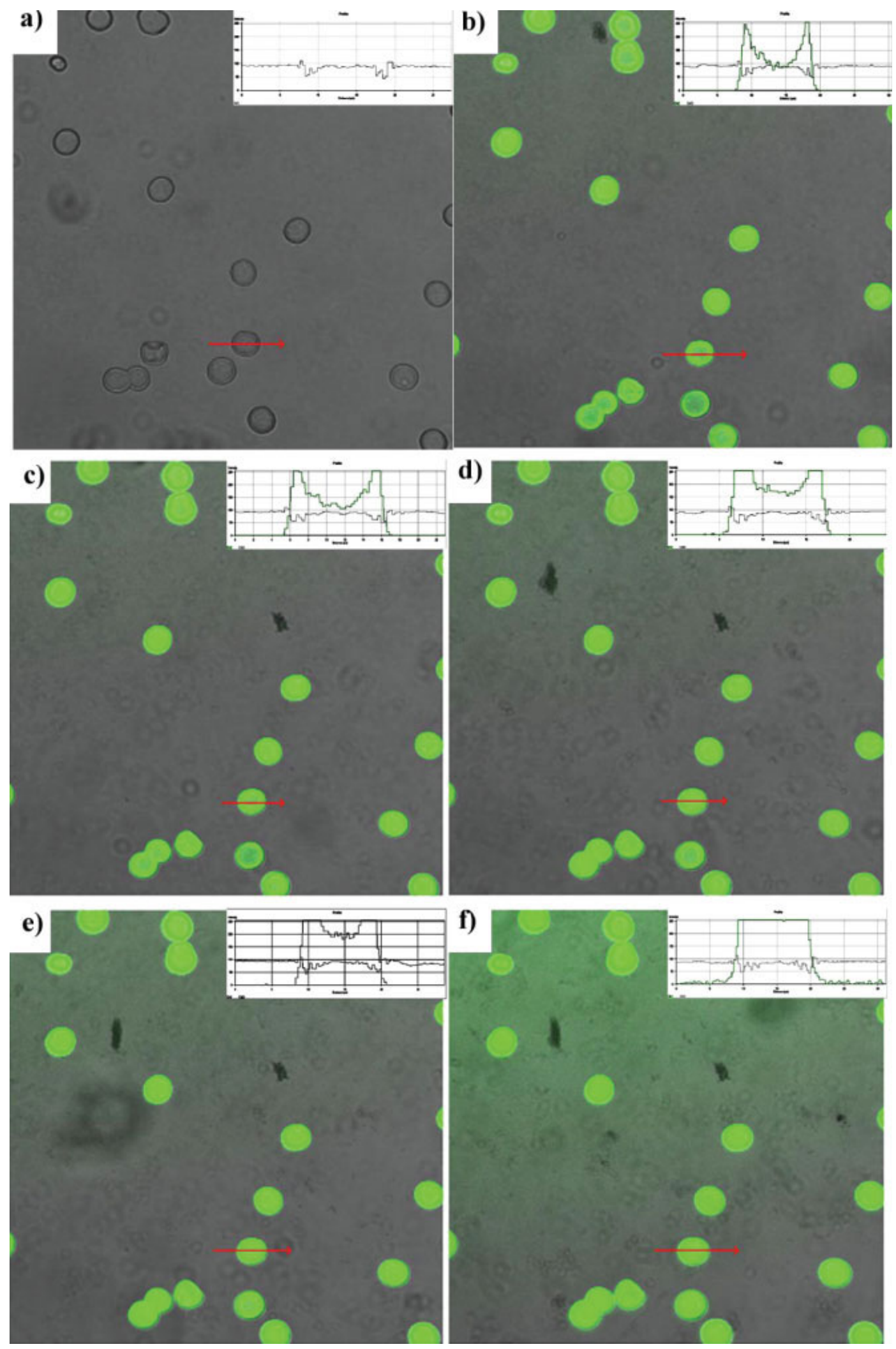

Figure 2. Representative CLSM images for loading FITC-lysozyme (molar ratio of the FITC to lysozyme is about 4:1) into hybrid capsules during real-time loading process: (a) $0 \mathrm{~min}$, (b) $60 \mathrm{~s}$, (c) $180 \mathrm{~s}$, (d) $360 \mathrm{~s}$, (e) $600 \mathrm{~s}$, (f) $900 \mathrm{~s}$; inset picture shows line-scan of fluorescence intensity for a single microcapsule. The concentration of FITC-lysozyme is $40 \mu \mathrm{g} / \mathrm{mL}$. [Color figure can be viewed in the online issue, which is available at www.interscience.wiley.com.] 


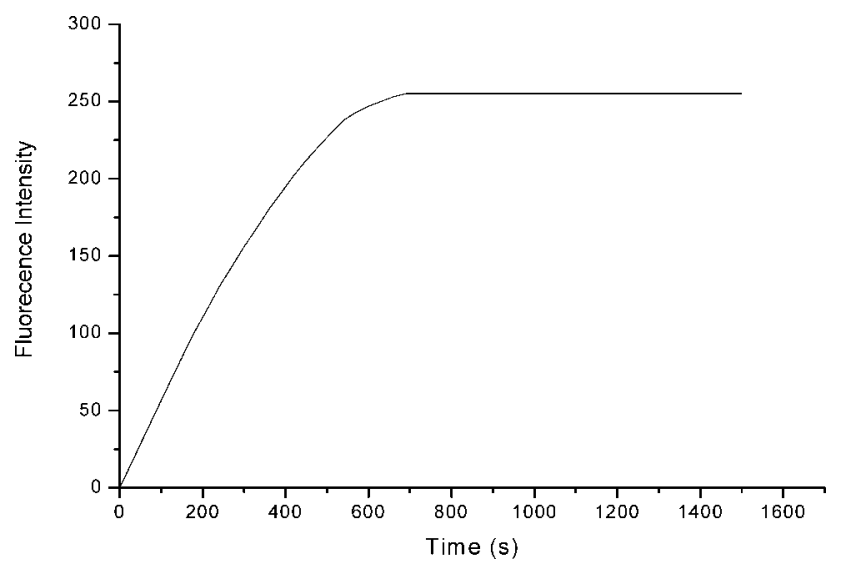

Figure 3. The dynamic process of induced deposition of FITC-lysozyme.

that in outside. The dynamics curve of induced deposition showed that the fluorescent intensity in the selected capsule came up to the maximum value after about $700 \mathrm{~s}$ (Fig. 3). Consequently, it was rather rapid for FITC-lysozyme to be entrapped into "micelles enhanced" capsules.

SFM was used to observe the top view of the capsules in dried state and determine the thickness of the capsule wall before and after incubation (Fig. 4). The wall of dry hybrid PE capsule came up to $110 \pm$ $20 \mathrm{~nm}$ [Fig. 4(a)]. As reported by Mohwald and coworkers, the thickness of each PE bi-layer is about $5 \mathrm{~nm} .{ }^{20}$ The wall of hybrid capsules is significantly thicker than that of the conventional PE capsules with the same six deposition bilayers ( $30 \mathrm{~nm})$. SFM image in Figure $4(\mathrm{~b})$ is the top view of the hybrid capsules after incubation. The wall of dry capsules reached $180 \pm 20 \mathrm{~nm}$. The difference between the capsule wall before and after incubation came up to about $70 \mathrm{~nm}$. Supposing that a lysozyme monolayer adsorbed on the outside PSS layer is $5 \mathrm{~nm}$, the amount of lysozyme adsorbed on a capsule can be calculated as $\int_{0}^{5} f(x) \mathrm{d} x .(f(x)$ is a function, which denotes lysozyme mass adsorbed on the unit thickness of surface of hybrid capsules, $\mathrm{ng} / \mathrm{nm}$; $\mathrm{d} x$ is differential variable, which denotes the thickness of lysozyme adsorbed on the surface of capsule, $\mathrm{nm}$ ). In a limited scope, $f(x)$ can be supposed as a constant $N(\mathrm{ng} / \mathrm{nm})$. Thus the adsorbed lysozyme should be less than $5 \mathrm{~N}$, and the amount of encapsulated lysozyme can be considered as about $65 \mathrm{~N}$. The ratio of lysozyme encapsulated inside the capsule to that adsorbed on the capsule surface can be evaluated as about 13:1, which indicated that more than $90 \%$ of lysozyme was encapsulated into the capsules. The results of SFM suggested that water soluble protein $(\mathrm{pI}>7)$ can be encapsulated into this novel kind of capsules with high efficiency.

The lysozyme concentration inside the capsule was further quantified by UV-Vis spectroscopy. Cap- sule concentration was $1.7 \times 10^{7} / \mathrm{mL}$ as measured by flow cytometer. The mean size of the hybrid capsules is $9.8 \mu \mathrm{m}$ by size analyzer. According to Eq. (1), the mean concentration inside the capsule interior can be calculated. So the mean amount of encapsulated substances for a single capsule can be evaluated as well. When the concentration of incubation solution was below $50 \mu \mathrm{g} / \mathrm{mL}$, the supernatant concentration was beyond the UV-Vis lowest detection limit. So it could be considered that almost all the FITC-lysozyme was all entrapped into capsules under this condition (this point can be illuminated in Fig. 2). As shown in Figure 5, with the concentration of incubation solution increasing from 20 to $2000 \mu \mathrm{g} / \mathrm{mL}$, the concentration inside the capsule interior increased strikingly from about 0.6 to $46 \mathrm{mg} /$ $\mathrm{mL}$. And the concentration of lysozyme inside capsule is about 80 times higher than that of bulk solution outside capsules. The same experiment was designed for entrapping FITC-triptoreline $\left(M_{\mathrm{w}}=\right.$ 1131, a positively charged water soluble peptide used as efficacious candidate for prostate cancer in clinic) into "micelles enhanced" PE capsules, the result was similar to that of lysozyme and the concentration inside the capsule interior increased from 0.6 to $41 \mathrm{mg} / \mathrm{mL}$.

FRAP technique was employed to demonstrate that lysozyme was deposited in a complexed form (Fig. 6). FITC-lysozyme was used as a detecting probe. Three capsules possessing induced deposition were selected. After photobleaching the spots shown in Figure 6(a), only the bleached spot in capsules became dark, which suggested that there was no mass exchange within the capsule interior. Other three capsules were selected as a control group. The fluorescence recovery profiles [Fig. 6(b)] demonstrated that the fluorescence intensity decreased a little for both the three detected capsules and the three control capsules. The decrease of fluorescence intensity for the control group can be attributed to the self-bleaching. The slopes of the bleaching profiles and control profiles were nearly consistent. Consequently, it could be considered that no recovery of fluorescence intensity occurs inside the capsules, which in turn suggested that there was no mass exchange inside the capsule interior. These results indicated that the deposited substances should be in a complexed form.

\section{The mechanism of induced encapsulation effect of "micelles enhanced" PE capsules}

A brief procedure for induced deposition process is illustrated in Scheme 1. On one side, the negatively charged PS- $b$-PAA based micelles interact with the most inner positively charged PAH layer to re- 

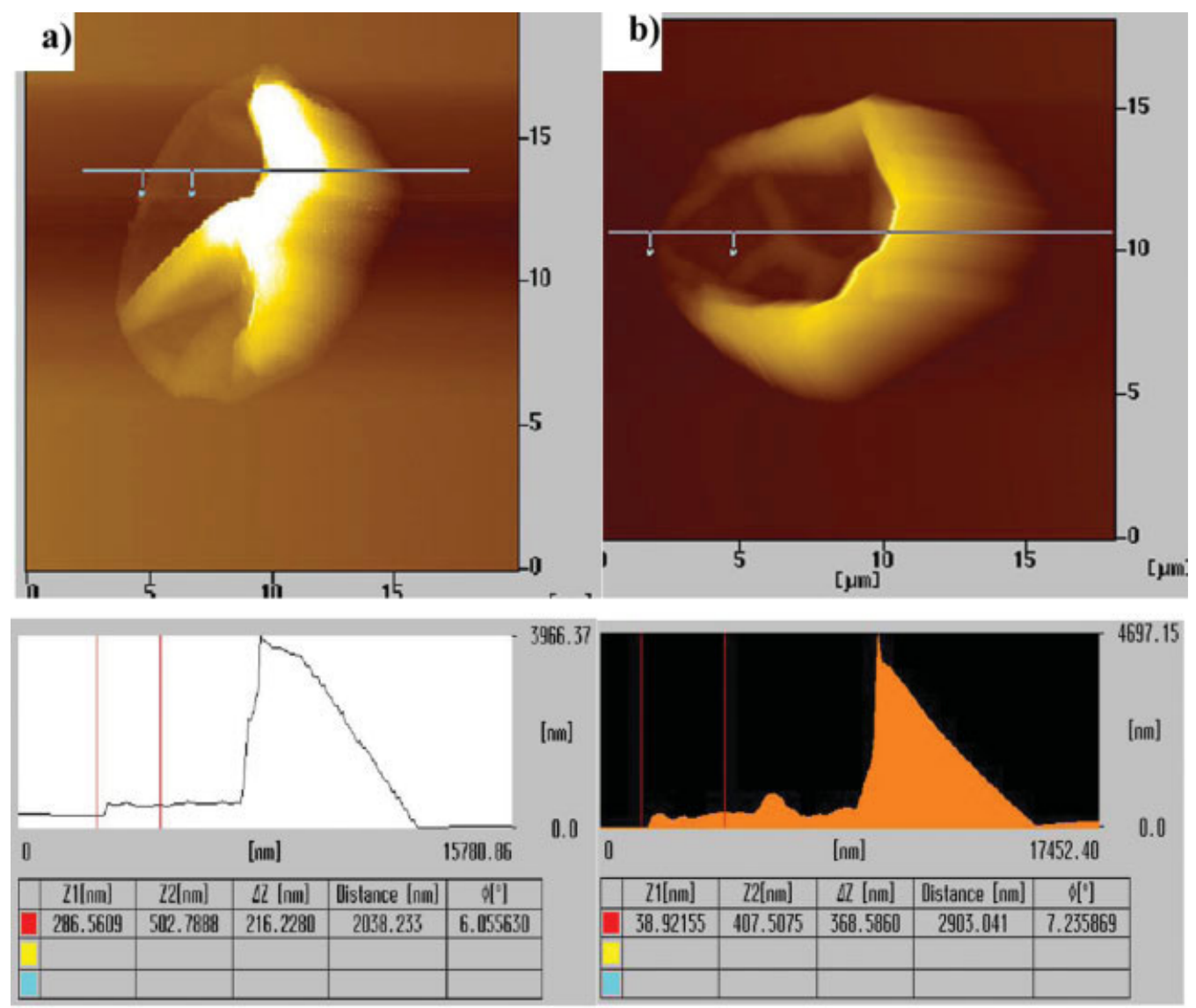

\section{$\Delta \mathrm{H}=\Delta \mathrm{Z} / 2=109 \pm 20 \mathrm{~nm}$}

$\Delta \mathrm{H}=\Delta \mathrm{Z} / 2=180 \pm 20 \mathrm{~nm}$

Figure 4. SFM images of hybrid microparticles. (a) individual capsule before incubation; (b) individual capsules after incubation. $\Delta H$ is the thickness of the capsule wall. [Color figure can be viewed in the online issue, which is available at www.interscience.wiley.com.]

construct the capsule wall. On the other side, a large number of stable negatively charged micelles provides huge negatively charged surfaces [Scheme 1(a)], which can be served as the driving force to induce the positively charged water-soluble substances into the capsule interior [Scheme 1(b,c)].

\section{Study on the encapsulation of ampholyte into capsules}

Considering proteins with isoelectric point below 7 can be changed into positively charged PEs when $\mathrm{pH}$ value of environment is below isoelectric point, experiments were also performed to investigate the encapsulation of this type of proteins into PE capsules. FITC-albumin ( $\mathrm{pI}=4.7)$ was used as the model protein to observe the change of encapsulation with the change of protein surface potential (Fig. 7). In pure water $(\mathrm{pH}$ is about 6.4), albumin is negatively charged PE, and it cannot be entrapped inside the capsules [Fig. 7(a)]. However, albumin will be positively charged when it is dissolved in acidic solution
( $\mathrm{pH}=3$ for instance). In this case, induced deposition occurred as shown in Figure 7(b-f). The fluorescence intensity inside the capsules at $450 \mathrm{~s}$ came up

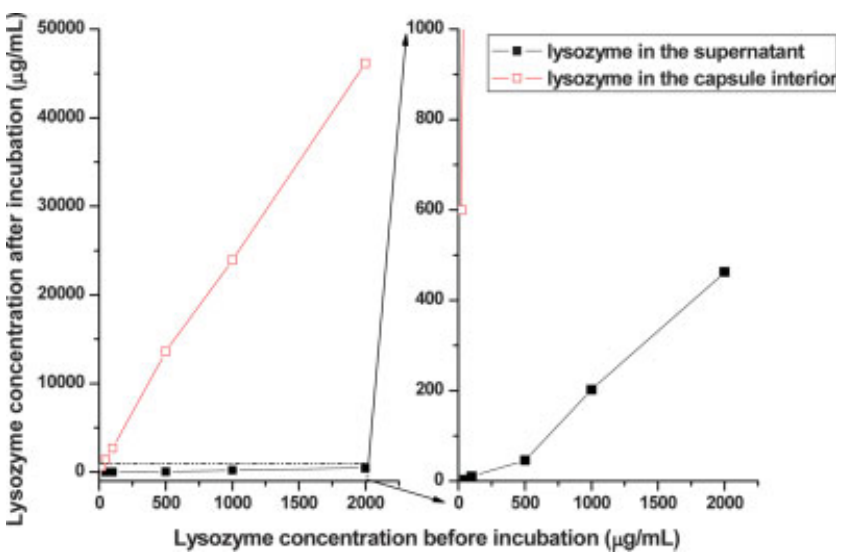

Figure 5. Concentration dependent loading of FITC-lysozyme into capsules. [Color figure can be viewed in the online issue, which is available at www.interscience.wiley. com.] 


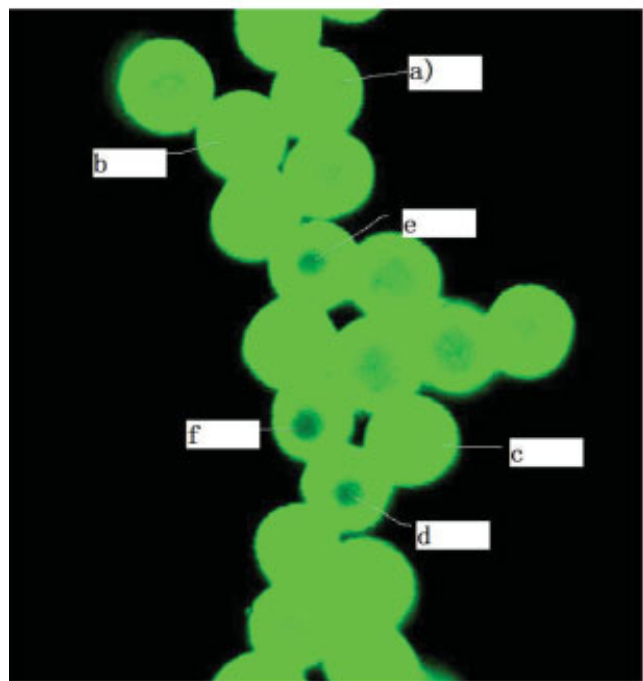

a)

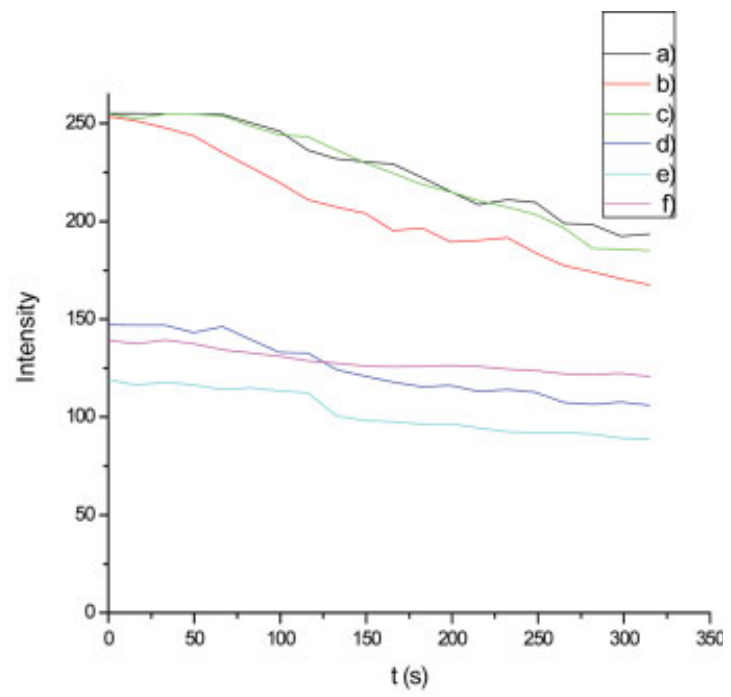

b)

Figure 6. Fluorescence recovery after photobleaching (FRAP) analysis demonstrates that FITC-lysozyme only self-deposited in an aggregated or complexed form, rather than in their free state. FITC-lysozyme was employed as a detecting probe. Three capsules $\mathrm{d}, \mathrm{e}, \mathrm{f}$ that exhibit induced deposition effect were bleached simultaneously at the marked spots in (a). After bleaching, the capsules a, b, c were selected as control groups, the fluorescence intensity profiles (b) were obtained. [Color figure can be viewed in the online issue, which is available at www.interscience.wiley.com.]

to peak value of the instruments. This result clearly demonstrated FITC-albumin, a negatively charged PE in neutral solution, could be rapidly entrapped into "micelles enhanced" PE capsules by modulating the surface potential of albumin molecules.

In addition, evidences showed that negatively charged substances such as sodium fluorescein, albumin could not enter the capsule interior, independent of molecule weight (unpublished data), whereas positively charged water-soluble substances could be efficiently entrapped without any additional force. This process occured due to the presence of PS- $b$ PAA based micelles inside the capsule interior. These micelles provide a huge negatively charged surface, which serves as a driving force to induce positively charged substances into the capsule interior. The capsule wall seems like semipermeable ion exchange membrane, allowing passage of certain positively charged water soluble proteins or peptides, while acting as a barrier to the negatively charged. Additionally, water soluble proteins or peptides are ampholytes, and the electrical properties of ampholytes can be modulated to positive potential by modulating the $\mathrm{pH}$ value of solution below the corresponding isoelectric point. Accordingly, this kind of hybrid capsules can efficiently entrap water soluble ampholytes with appropriate molecule weight as well.

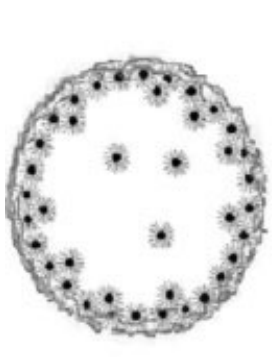

a)

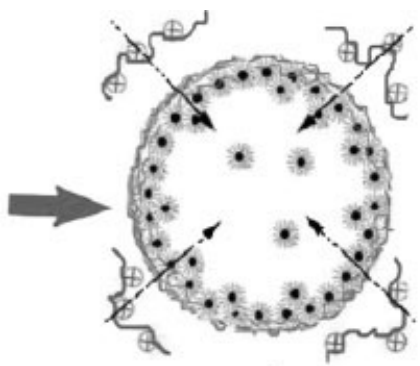

b)

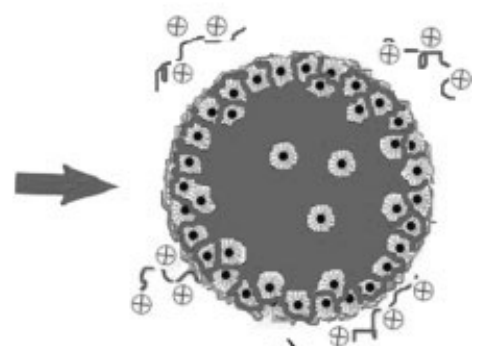

c)

Scheme 1. Schematic illustration of the spontaneous deposition mechanism based on the formation of the super-micelles network congeries during the core dissolution process: (a) formation of "micelle-enhanced" interior wall and micelle/Ca" complex in the capsule, (b) capsules exposed in the positively charged species, (c) spontaneous deposition. 


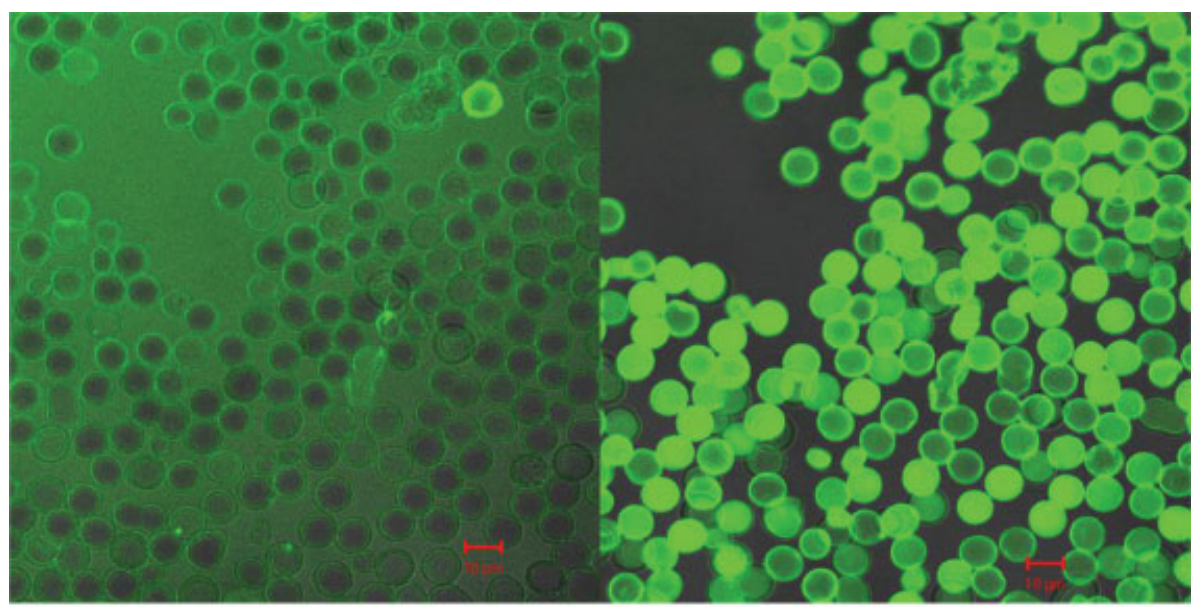

a)

b)

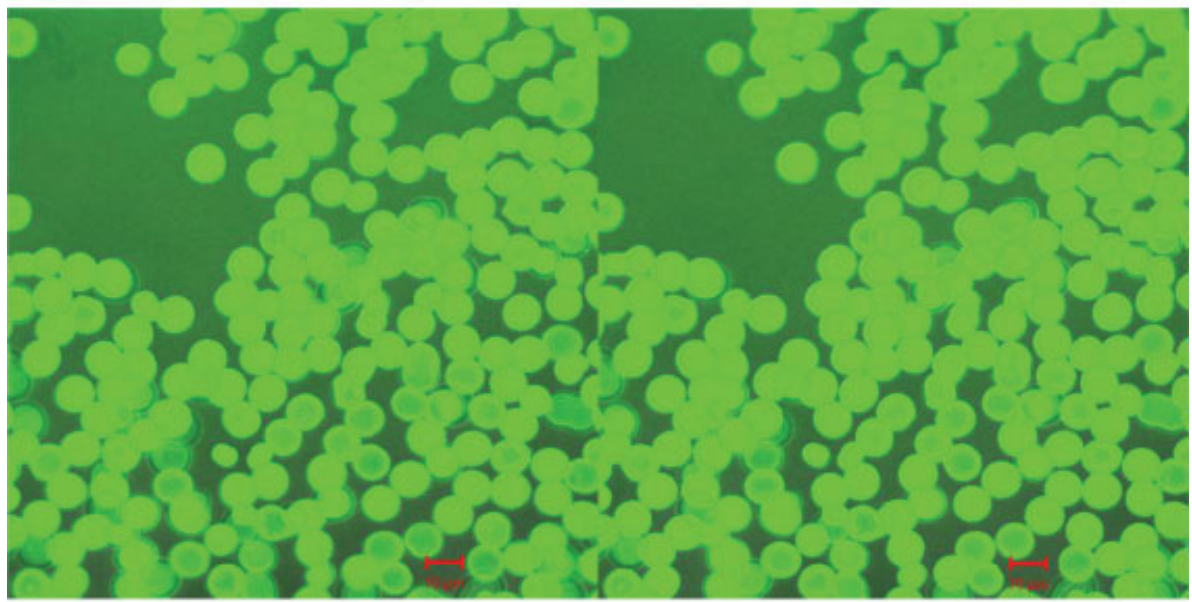

c)

d)

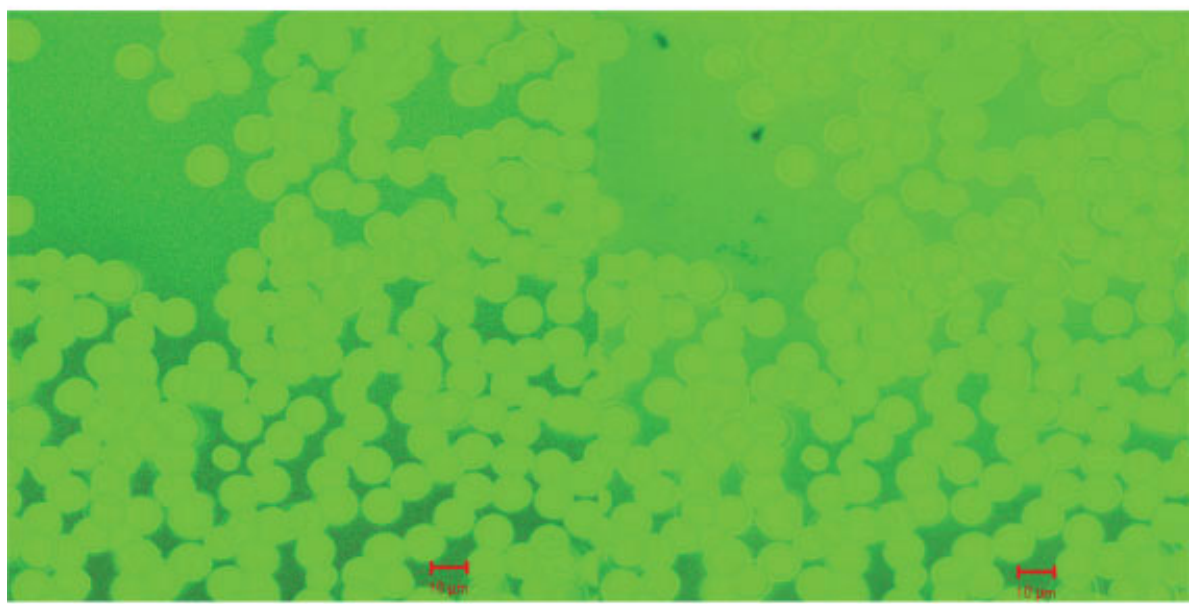

e)

f)

Figure 7. CLSM was used to observe FITC-albumin encapsulation inside hybrid capsules in different $\mathrm{pH}$ surrounding. FITC-albumin ( $\mathrm{pI}=4.9$ ) was used as fluorescence probe. (a) FITC-albumin cannot be entrapped into the capsule in pure water ( $\mathrm{pH}$ value of bulk solution is about $6.4>\mathrm{pI}$ ), time was $0 \mathrm{~s}$. (b) FITC-albumin can be deposited into capsules in acidic solution ( $\mathrm{pH}$ value of bulk solution is $3<\mathrm{pI}$ ), time is $60 \mathrm{~s}$. (c) time is $150 \mathrm{~s}$. (d) time is $300 \mathrm{~s}$. (e) time is $450 \mathrm{~s}$. (f) time is $660 \mathrm{~s}$. The concentration of FITC-albumin is $40 \mu \mathrm{g} / \mathrm{mL}$. [Color figure can be viewed in the online issue, which is available at www.interscience.wiley.com.] 


\section{CONCLUSION}

In conclusion, by introducing functional templates for fabricating PE capsules by LbL technique, novel "micelles enhanced" PE multilayer capsules were obtained, which could entrap peptides, proteins, and other ampholytes with high efficiency. The size of these novel capsules ranges from about 1 to $10 \mu \mathrm{m}$ according to the corresponding templates used. ${ }^{17}$ The method of preparing the PE capsules is easy, convenient, and reproducible. These novel capsules might find their way in drug delivery system, enzyme fixation, bioreactor, and biosensor. Additionally, since the wall structure has a great effect on the encapsulation and release behaviors of these PE capsules, further study on the wall structure and its influence on the other physicochemical properties is under way, and the related results will be reported in near future.

The authors thank Prof. Chen Yiyong (Zhejiang University) and Prof. Donglu Shi (University of Cincinnati) for their advice to this study. The authors also thank Dr. Zhang Le for his help in CLSM observation.

\section{References}

1. Caruso F, Rachel A, Möhwald H. Nanoengineering of inorganic and hybrid hollow spheres by colloidal templating. Science 1998;282:1111-1114.

2. Donath E, Sukhorukov GB, Caruso F, Davis SA, Möhwald H. Novel hollow polymer shells by colloed-templated assembly of polyelectroytes. Angew Chem Int Ed 1998;37:2201-2205.

3. Gao CY, Möhwald H, Shen JC. Soluble microcapsules assembled stepwise from weak polyelecrolytes using aciddecomposable cores. Adv Mater 2003;15:930-933.

4. Zhang YJ, Guan Y, Yang SG, Xu J, Han CC. Fabrication of hollow capsules based on hydrogen bonding. Adv Mater 2003;15:832-835.

5. Moya S, Donath E, Sukhorukov GB, Auch M, Baumler H, Lichtenfeld H, Möhwald H. Lipid coating on polyelectrolyte surface modified colloidal particles and polyelectrolyte capsules. Macromolecules 2000;33:4538-4544.

6. Dai ZF, Voigt A, Leporatti S, Donath E, Dahne L, Hartmann J, Donath E, Möhwald H. Layer-by-layer self-assembly of poly- electrolyte and low molecular weight species into capsules. Adv Mater 2001;13:1339-1342.

7. Sukhorukov GB, Dahne L, Hartmann J, Donath E, Möhwald $\mathrm{H}$. Controlled precipitation of dyes into hollow polyelectrolyte capsules based on colloids and biocolloeds. Adv Mater 2000;12:112-115.

8. Dai ZF, Dahne L, Möhwald H, Tiesch B. Novel capsules with high stability and controlled permeability by hierarchic templating. Angew Chem Int Ed 2002;41:4019-4022.

9. Caruso F, Trau D, Mohwald H, Renneberg R. Enzyme encapsulation in layer by layer engineered polymer multilayer capsules. Langmuir 2000;16:1485-1488.

10. Qiu XP, Leporatti S, Donath E, Mohwald H. Studies on the drug release properties of polysaccharide multilayers encapsulated ibuprofen microparticles. Langmuir 2001;17:53755380 .

11. Shchukin DG, Patel AA, Sukhorukov GB, Lvov YM. Nanoassembly of biodegradable microcapsules for DNA encasing. J Am Chem Soc 2004;126:3374-3375.

12. Dähne L, Leporatti S, Donath E, Möhwald H. Fabrication of micro reaction cages with tailored properties. J Am Chem Soc 2001;123:5431-5436.

13. Ibarz G, Dähne L, Donath E, Möhwald H. Smart micro- and nanocontainers for storage, transport, and release. Adv Mater 2001;13:1324-1327.

14. Sukhorukov GB, Antipov AA, Voigt A, Donath E, Möhwald $\mathrm{H}$. $\mathrm{pH}$-controlled macromolecule encapsulation in and release from polyelectrolyte multilayer nanocapsules. Macromol Rapid Commun 2001;22:44-46.

15. Gao CY, Liu XY, Shen JC, Möhwald H. Spontaneous deposition of horseradish peroxidase into polyelectrolyte multilayer capsules to improve its activity and stability. Chem Commun 2002;1928-1929.

16. Peyratout CS, Dähne L. Tailor-made polyelectrolyte microcapsules: From multilayers to smart containers. Angew Chem Int Ed 2003;15:1722-1726.

17. Li XD, Hu QL, Yue LH, Shen JC. Synthesis of size-controlled acid-resistant hybrid calcium carbonate microparticles as templates for fabricating 'micelles enhanced' polyelectrolyte capsules by the LbL technique. Chem-Eur J 2006;12:57705778 .

18. Volodkin DV, Petrov AI, Prevot M, Sukhorukov GB. Matrix polyelectrolyte microcapsules: New system for macromolecule encapsulation. Langmuir 2004;20:3398-3406.

19. Volodkin DV, Larionova NI, Sukhorukov GB. Protein encapsulation via porous $\mathrm{CaCO}_{3}$ microparticles templating. Biomacromolecules 2004;5:1962-1972.

20. Estrela-Lopis I, Leporatti S, Moya S, Brandt A, Donath E, Möhwald H. SANS studies of polyelectrolyte multilayers on colloidal templates. Langmuir 2002;18:7861-7866. 